\title{
CINÉTICA DA ETANÓLISE DO ÓLEO DE GIRASSOL
}

\author{
T. P. V. B. DIAS ${ }^{1}$, B. H. LUNELLI ${ }^{2}$, P. MIELKE NETO ${ }^{1}$, L. A. FOLLEGATTI-ROMERO ${ }^{2}$ e \\ A. J. A. MEIRELLES ${ }^{1}$ \\ ${ }^{1}$ Universidade Estadual de Campinas, Departamento de Engenharia de Alimentos \\ ${ }^{2}$ Universidade Estadual de Campinas, Faculdade de Engenharia Química \\ E-mail para contato: telmaporcina@yahoo.com.br
}

\begin{abstract}
RESUMO - Dados cinéticos da transesterificação do óleo de girassol com etanol para a produção de biodiesel, utilizando etóxido de sódio como catalisador, foram determinados experimentalmente e modelados com base em um modelo cinético reversível. Os ensaios experimentais foram realizados a diferentes temperaturas $(308,15,323,15$ e $338,15 \mathrm{~K})$, com uma razão molar de 9:1 (etanol:triglicerídeo) e $1 \%$ (em peso) de etóxido de sódio. As reações foram realizadas por um período de $120 \mathrm{~min}$, sendo retiradas amostras do processo de reação em tempos determinados. As amostras foram análisadas por cromatografia líquida de alta performance por exclusão de tamanho (HPSEC) para a determinação da concentração dos compostos. Os modelos matemático e cinético desenvolvidos para o processo de transesterificação foram simulados com o auxílio de um software escrito em FORTRAN. A partir das simulações realizadas foi possível determinar as constantes de velocidade de reação e as energias de ativação para o processo de transesterificação do óleo de girassol.
\end{abstract}

\section{INTRODUÇÃO}

O biodiesel é um tipo de combustível renovável, que é produzido principalmente a partir de óleos vegetais e gorduras animais, sendo de enorme interesse como combustível alternativo. O biodiesel é seguro, renovável, não tóxico e biodegradável (Kiss et. al., 2006). Este biocombustível tem se mostrado como um bom sucessor do diesel fóssil e uma alternativa interessante para a diversificação da matriz energética mundial (Sonnino, 1994). Os processos de esterificação e transesterificação são algumas das rotas utilizadas na sua produção (Meneghetti et. al., 2006).

A transesterificação de triglicerídeos, metílica ou etílica, utilizando catalisadores alcalinos ou ácidos, é atualmente o caminho mais utilizado para produção de biodiesel (Souza et. al., 2007). Variados métodos de análise podem ser empregados para avaliar o efeito dos parâmetros de reação sobre a pureza e rendimento do biodiesel, uma vez que eles são mais rápidos e mais baratos do que outros métodos e permitem a otimização de várias variáveis que influenciam o processo de produção (Montegomery, 1997).

O estudo cinético da etanólise básica de óleos vegetais ainda é pouco reportada pela literatura, assim como os dados de equilíbrio de compostos presentes durante a produção do 


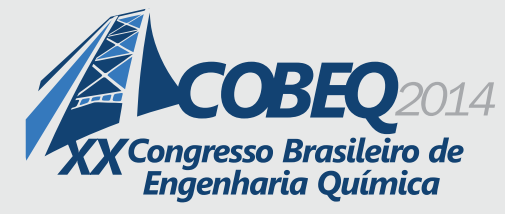

19 a 22 de outubro de 2014

Florianópolis/SC

biodiesel. Estas informações são importantes para estabelecer condições de operação do sistema com quantidades ótimas dos reagentes e temperaturas nas quais a reação deve ser processada. Além disso, com a busca cada vez maior por fontes de energia renováveis e menos poluidoras, a obtenção de dados que elucidam os detalhes desse processo de produção é de grande importância. Este trabalho de pesquisa visa à investigação da cinética reacional na produção de biodiesel por transesterificação homogênea básica do óleo de girassol.

\section{MATERIAL E MÉTODOS}

Para a realização deste trabalho foi utilizado óleo de girassol refinado (Bunge Alimentos SA), etóxido de sódio (99 \% - Sigma Aldrich), etanol anidro (99,5 \% - Merck) e Tetrahidrofurano (THF) grau HPLC (99,5 \% - Tedia). A composição do óleo utilizado foi determinada em ácidos graxos através de cromatografia gasosa, segundo metodologia proposta por Basso et al. (2012).

A produção de biodiesel a partir da transesterificação do óleo de girassol foi realizada em três diferentes temperaturas, a saber, $308,15,323,15$ e 338,15 K. Os ensaios foram realizados nas seguintes condições: massa de óleo/triacilgliceróis de aproximadamente 100 gramas (capacidade máxima do equipamento); rotação do agitador de aproximadamente $400 \mathrm{rpm}$; tempo de reação de 120 minutos; quantidade de catalisador de 1,0\% (em peso do triacilglicerol) e uma razão molar de etanol:óleo de 9:1. As condições operacionais escolhidas para o processo de transesterificação do óleo de girassol foram baseadas em dados disponíveis na literatura (Likozar, 2014; Marjanovic, 2010; Ahiekpor, 2010).

A reação foi realizada numa célula de equilibrio líquido-líquido de $200 \mathrm{~mL}$. As células são encamisadas, com saída e entrada para a conexão de um banho termostático, além disto, estas células apresentam duas pequenas aberturas transversais (superior e inferior) que são vedadas por septos de silicone e são utilizadas para retirada de amostras. As células foram conectadas em um banho termostático (Cole Parmer, modelo 12101-55, USA) para o controle de temperatura e vedadas com tampa de teflon que possui uma entrada na qual é acoplado um termômetro digital (Alla, modelo -50 a $200{ }^{\circ} \mathrm{C}$, França), utilizado para o monitoramento da temperatura do sistema.

A célula de vidro contendo o óleo ( 100 g) foi mantida sob agitação com auxílio de uma barra magnética e agitador (Ika Werke, modelo RH-KT/C, Alemanha) até atingir a temperatura desejada (cerca de 30 minutos). A mistura contendo o etóxido de sódio e etanol foi preparada e termostatizada separadamente, sendo posteriormente adicionada à célula, a partir da adição dos componentes da reação, o tempo passou a ser cronometrado. Durante a reação foram retiradas amostras $(\sim 0,5 \mathrm{~mL})$ da mistura em tempos pré-determinados, sendo estas amostras adicionadas imediatamnte em $10 \mathrm{~mL}$ de THF para interromper a reação. As amostras foram, posteriomente, filtradas em um filtro Millipore de 0,45 $\mu \mathrm{m}$ e mantidas sob refrigeração.

A quantificação dos produtos da reação de transesterificação foi realizada empregando a cromatografia líquida por exclusão de tamanho (High-Performance Size Exclusion Chromatography - HPSEC). A metodologia utilizada foi adaptada de Schoenfelder (2003), as 


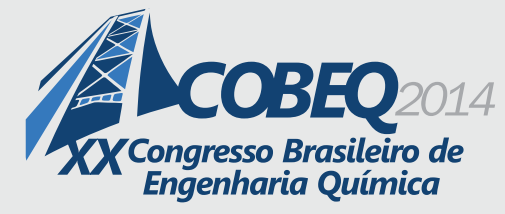

colunas propostas pelo método foram substituídas por três colunas Phenogel, 5 microns, de 50A, 100A e 500A de 300x7,8 mm, da Marca PHENOMENEX. O resultado obtido no cromatograma é em frações mássicas de cada componente, as quais são calculadas mediante as áreas dos picos de cada componente dividida pela soma total das áreas de todos os componentes.

\section{ESTIMATIVA DOS PARÂMETROS CINÉTICOS}

Os modelos matemático e cinético desenvolvidos para o processo de transesterificação foram simulados com o auxílio de um software escrito em FORTRAN. A integração das equações diferenciais ordinárias do modelo foi realizada utilizando o método de Runge-Kutta de $4^{\mathrm{a}}$ ordem. Os parâmetros cinéticos do modelo foram estimados a partir dos dados experimentais obtidos. Para a estimativa dos parâmetros cinéticos, foi utilizada a técnica de otimização por algoritmo genético (Genetic Algorithm - GA, Holland, 1992). Para tanto, foi utilizado o otimizador desenvolvido por Carrol (1996), o qual foi adaptado ao software de simulação (Victorino, 2005, Lunelli et al., 2011). Para minimizar a diferença entre os dados preditos pelo modelo (simulado) e os dados experimentais, foi utilizada a seguinte função objetivo:

$$
F_{O B J}=\sum_{i=1}^{I} \sum_{k=1}^{K}\left(\frac{C_{s i m_{i, k}}-C_{\exp _{i, k}}}{C_{\exp } \max }\right)^{2}
$$

Com $i$ variando ao longo do tempo e $C k$ se referindo às concentrações dos componentes da reação.

O modelo cinético utilizado na simulação foi baseado no modelo proposto por Noureddini \& Zhu (1997). O processo de produção de biodiesel utilizando etanol ocorre em uma única fase (regime pseudo-homogêneo), sendo assim determinado unicamente pela cinética de reação e pode ser representado pelas seguintes reações:

$$
\begin{aligned}
& T G+E T \underset{\mathrm{k}_{2}}{\stackrel{\mathrm{k}_{1}}{\rightleftarrows}} D G+E E \\
& D G+E T \underset{\mathrm{k}_{4}}{\stackrel{\mathrm{k}_{3}}{\rightleftarrows}} M G+E E \\
& M G+E T \underset{\mathrm{k}_{6}}{\stackrel{\mathrm{k}_{5}}{\rightleftarrows}} G L+E E
\end{aligned}
$$

Onde, TG, DG, MG, ET, GL, EE, correspondem aos componentes tri-, di- e monoglicerídeo, etanol, glicerol e éster etílico, respectivamente e $\mathrm{k}_{1}$ a $\mathrm{k}_{6}$ são as constantes da taxa de reação.

Os valores das constantes da taxa de reação $\left(k_{i}\right)$ foram determinados a partir da regressão linear das concentrações de reagentes e produtos ao longo do tempo, obtidas experimentalmente e, utilizados como estimativa inicial no ajuste dos parâmetros cinéticos do modelo. De posse dos valores das constantes cinéticas $\left(k_{i}\right)$, as energias de ativação $(E a)$ e os fatores pré-exponenciais $\left(k_{0}\right)$ foram determinados, através da linearização da Equação de Arrhenius (3). 


$$
\ln (k)=-\left(\frac{E_{a}}{R}\right) \times \frac{1}{T}+\ln \left(k_{0}\right)
$$

\section{RESULTADOS E DISCUSSÃO}

\subsection{Caracterização da matéria-prima}

A composição do óleo de girassol refinado em ácidos graxos e do biodiesel em ésteres de ácidos graxos utilizado neste trabalho é apresentada na Tabela 1. De posse destes resultados, foi feita a estimativa da provável composição do óleo de girassol em TAG's através do método computacional estatístico recomendado por Antoniosi Filho et al. (1995), a partir da composição molar em ácidos graxos. Este método computacional se baseia nas teorias da distribuição "casual" e "1,3-casual 2-casual". A teoria da distribuição "casual" diz que os ácidos graxos se encontram distribuídos ao acaso, estatisticamente, entre as três posições da molécula do glicerol. Para calcular a composição provável em triacilgliceróis, dois aspectos foram considerados, a saber, 1. As composições dos isômeros trans foram somadas aos seus respectivos isômeros cis. 2. Os triacilgliceróis que apresentaram valores menores que $0,5 \%$ foram ignorados.

Tabela 1 - Composição de ácidos graxos do óleo de girassol

\begin{tabular}{cccc}
\hline Ácido Graxo & Símbolo & $\mathrm{Cx}: \mathrm{y}^{\mathrm{a}}$ & $100 \mathrm{w}$ \\
\hline Láurico & $\mathrm{L}$ & $\mathrm{C} 12: 0$ & 0,06 \\
Mirístico & $\mathrm{M}$ & $\mathrm{C} 14: 0$ & 0,02 \\
Palmítico & $\mathrm{P}$ & $\mathrm{C} 16: 0$ & 6,30 \\
Palmitoleico & $\mathrm{Po}$ & $\mathrm{C} 16: 1$ & 0,09 \\
& & $\mathrm{C} 16: 1 \mathrm{t}^{\mathrm{c}}$ & 0,03 \\
Margárico & $\mathrm{Ma}$ & $\mathrm{C} 17: 0$ & 0,03 \\
& $\mathrm{Mg}$ & $\mathrm{C} 17: 1$ & 0,04 \\
Esteárico & $\mathrm{S}$ & $\mathrm{C} 18: 0$ & 3,31 \\
Oleico & $\mathrm{O}$ & $\mathrm{C} 18: 1$ & 35,98 \\
Linoleico & $\mathrm{Li}$ & $\mathrm{C} 18,2$ & 52,75 \\
& & $\mathrm{C} 18: 2 \mathrm{t}^{\mathrm{c}}$ & 0,11 \\
Linolênico & $\mathrm{Le}$ & $\mathrm{C} 18: 3$ & 0,78 \\
Araquídico & $\mathrm{A}$ & $\mathrm{C} 20: 0$ & 0,30 \\
Gadoleico & $\mathrm{Ga}$ & $\mathrm{C} 20: 1$ & 0,22 \\
\hline
\end{tabular}

${ }^{a}$ Cx:yonde $x=$ número de carbonos e $\mathrm{y}=$ número de duplas ligações. ${ }^{b} \mathrm{M}=$ massa molar. ${ }^{c}$ isômero trans.

A massa molar média para o óleo de girassol foi calculada a partir da ponderação das massas molares dos triacilgliceróis que compõem o óleo e o valor obtido foi de 877,66 g.mol ${ }^{-1}$. 


\subsection{Cinética do óleo de girassol}

Na Figura 1 é apresentada a curva típica da concentração dos componentes presentes na transesterificação do óleo de girassol na temperatura de 308,15 K. Pode-se verificar que houve consumo dos triacilgliceróis e do etanol, assim como a produção de ésteres etílicos, glicerina, mono- e diacilgliceróis.

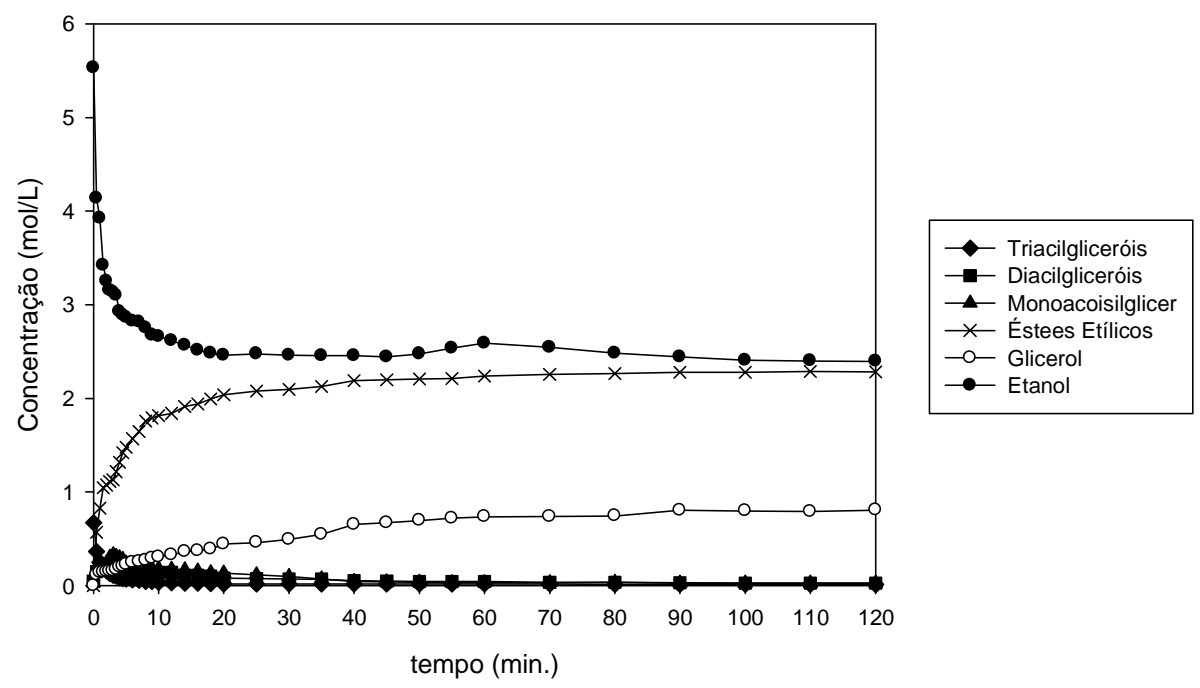

Figura 1 - Perfis de concentração da transesterificação do óleo de girassol a 308,15 K.

A primeira fase da transesterificação é controlada pela transferência de massa. Devido ao fato do óleo não ser miscível no álcool, esta fase é caracterizada por lentas taxas de reação, daí a importância de agitação nesta fase. Em geral, a primeira fase é mais rápida na reação de etanólise em relação à reação de metanólise, devido à diferença na solubilidade entre os álcoois. A segunda fase é rápida e é controlada pela cinética da reação, enquanto que a última fase é dominada pelo equilíbrio químico (Zhou, 2006).

A taxa de produção de ésteres etílicos na Figura 1 inicia com um aumento repentino seguido por uma menor taxa de produção quando a reação se aproxima do equilíbrio. Noureddine e Zhu (1997), observaram um padrão sigmoidal para a produção de ésteres metílicos, este padrão é constituído por uma velocidade lenta no início, seguida por um aumento repentino e, finalmente, uma taxa lenta novamente, especialmente a baixas temperaturas. Segundo os autores, a região controlada pela transferência de massa pode ser eliminada se a mistura for suficientemente agitada.

Conforme podemos observar na Figura 1, a região controlada pela transferência de massa quase desaparece. Assim, a agitação fornecida é suficiente para manter o sistema 


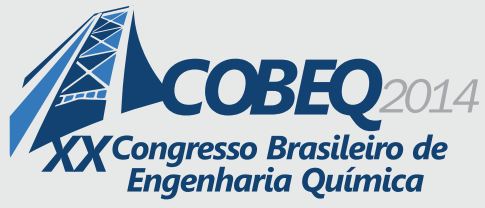

19 a 22 de outubro de 2014

Florianópolis/SC

homogêneo, mesmo na fase inicial da reação. Ahiekpor e Kuwornoo (2010) também observaram esse mesmo comportamento no estudo da etanólise do óleo de palma.

Como pode ser observado na Figura 1, a concentração de ésteres etílicos passou de 0,00 $\mathrm{mol} / \mathrm{L}$ para cerca de $1,48 \mathrm{~mol} / \mathrm{L}$ em 5 minutos de reação, sendo que em 20 minutos de reação sua concentração era de aproximadamente $2,00 \mathrm{~mol} / \mathrm{L}$, concentração máxima de ésteres etílicos obtido para este sistema a 308,15 K. Por outro lado, a concentração de triacilgliceróis diminui de seu valor inicial de $0,67 \mathrm{~mol} / \mathrm{L}$ para $0,1044 \mathrm{~mol} / \mathrm{L}$ em 3,50 minutos. As concentrações de monoe diacilgliceróis também aumentaram nos primeiros minutos de reação, atingindo um valor máximo, iniciando, em seguida, um período de redução lenta e gradual até o término da reação. De modo geral, pode-se afirmar que as concentrações de todos os componentes sofreram grandes alterações nos primeiros minutos de reação e a partir de 20 minutos tenderam a entra em equilíbrio nos sistemas estudados, atingindo quase o estado estacionário.

\subsection{Modelagem dos dados cinéticos para o óleo de girassol}

A partir da amostragem realizada em diferentes intervalos de tempo, foi possível quantificar as concentrações de mono-, di- e triacilgliceróis, glicerina, ésteres etílicos e etanol ao longo do tempo. Os perfis de concentrações obtidos foram utilizados na estimativa dos parâmetros cinéticos, bem como, as concentrações iniciais de cada reagente. A Figura 2 mostra os perfis de concentração dos dados experimentais e simulados para o processo de transesterificação do óleo de girassol a 308,15 K.

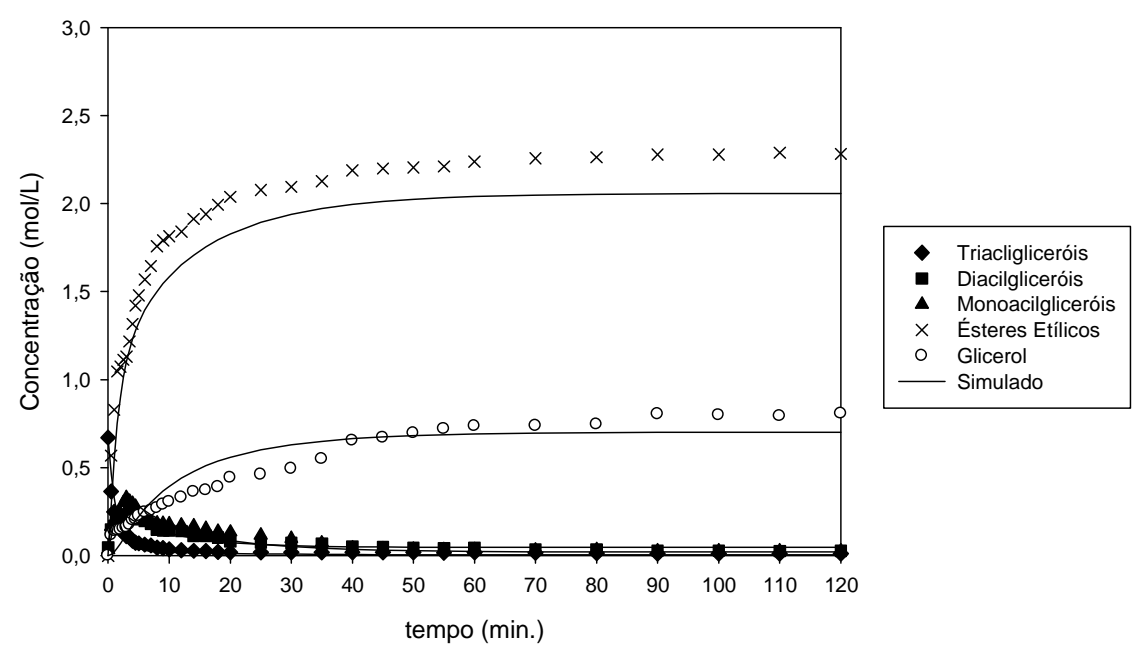

Figura 2 - Perfis de concentração experimental e simulado para o óleo de girassol a 308,15 K.

A Figura 3 mostra a dependência de $\ln (k)$ em função de $1 / T$, confirmando que a equação de Arrhenius (Equação 3) pode ser empregada na determinação das energias de ativação da reação de etanólise do óleo de girassol. 


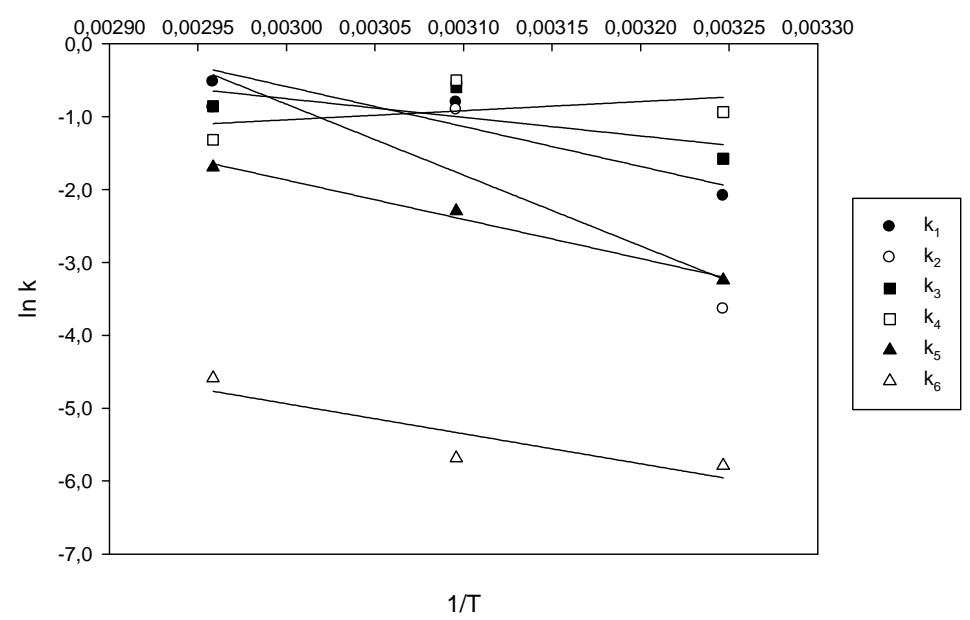

Figura 3 - Gráfico de Arrhenius para o óleo de girassol.

\section{CONCLUSÕES}

A etanólise de óleo de girassol foi estudada sob várias condições de reação utilizando como catalisador o etóxido de sódio, a fim de analisar os efeitos das condições de reação, tanto para o rendimento dos ésteres etílicos e a velocidade de reação, quanto para modelagem da cinética da reação de etanólise dos óleos estudados. Através da análise dos gráficos de concentração em função do tempo foi observado que as taxas de produção do éster etílico, iniciam com um aumento repentino seguido por uma etapa de velocidade de produção mais lenta, onde a reação se aproximou do equilíbrio. As constantes de velocidade de reação e as energias de ativação obtidas na estimativa dos parâmetros cinéticos, conseguiram reproduzir de forma satisfatória o sistema estudado, mostrando que o modelo cinético desenvolvido pode ser utilizado para descrever adequadamente o processo de transesterificação do óleo de girassol.

\section{AGRADECIMENTOS}

Os autores agradecem ao CNPq (483340/2012-0) e a FAPESP (2008/56258-8 e 2012/09646-8) pelo suporte financeiro.

\section{REFERÊNCIAS}

AHIEKPOR, J. C.; KUWORNOO, D. K.. Kinetics of palm kernel oil and ethanol transesterification. International Journal of Energy and Environment, v. 1, p. 1097 - 1108, 2010.

ANTONIOSI FILHO, N. R.; MENDES, O. L.; LANÇAS, F. M.. Computer Prediction of Triacylglycerol Composition of Vegetable Oils by HRGC. Chromatographia, v. 40, p. 557-562, 1995. 


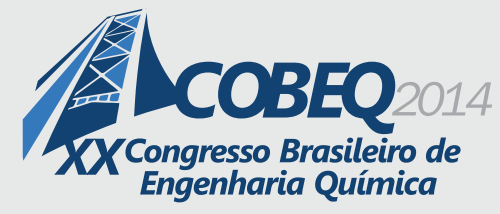

BASSO, R. C.; MEIRELlES, A. J. A.; BATISTA, E. A. C.. Liquid-liquid equilibrium of pseudoternary systems containing glycerol + ethanol + ethylic biodiesel from crambe oil (Crambe abyssinica) at $\mathrm{T} / \mathrm{K}=(298.2,318.2 \mathrm{e} 338.2)$ and thermodynamic modeling. Fluid Phase Equilibria, v. 333, p. 55-62, 2012.

CARROLL, D. L. Chemical laser modeling with genetic algorithms, AIAA Journal, 34, 338-346, 1996.

HOLLAND, J. H. Adaptation in natural and artificial systems: an introductory analysis with applications to biology, control and artificial intelligence. MIT Press, Cambridge, USA, 1992.

KISS, A. A. et al. Solid acid catalysts for biodiesel production - Towards sustainable energy. Advanced Synthesis \& Catalysis, v. 348, n. 1-2, p. 75-81, 2006.

LUNELli, B. H.; MELO, D. N. C.; MORAIS, E. R.; VICTORINO, I. R. S.; VASCO DE TOLEDO, E. C.; WOLF MACIEL, M. R.; MACIEL FILHO, R. Real-time optimization for lactic acid production from sucrose fermentation by Lactobacillus plantarum. 21st European Symposium on Computer Aided Process Engineering, p. 1396-1400, 2011.

MENEGHETTI, S. M. P. et al. Ethanolysis of castor and cottonseed oil: A systematic study using classical catalysts. Journal of the American Oil Chemists Society, v. 83, n. 9, p. 819-822, Sep 2006.

MONTGOMERY, D. C. Design and Analysis of Experiments. Wiley, New Yor,. v. 4, 1997.

NOUREDDINI, H.; ZHU, D. Kinetics of transesterification of soybean oil. Journal of the American Oil Chemists Society, v. 74, n. 11, p. 1457-1463, 1997.

SCHOENFELDER, W., European Journal of Lipid Science and Technology, v. 105, p. 45-48, 2003.

SONNINO, A. Agricultural biomass production is an energy option for the future. Renewable Energy, v. 5, n. 5-8, p. 857-865, 1994.

SOUZA, A. G. et al. Thermal and kinetic evaluation of cotton oil biodiesel. Journal of Thermal Analysis and Calorimetry, v. 90, n. 3, p. 945-949, 2007.

VICTORINO, I. R. S. Otimização de um reator industrial de produção de álcool cíclico utilizando algoritmos genéticos. Tese (Doutorado). Faculdade de Engenharia Química, UNICAMP, Campinas, SP, 2005.

ZHOU, W.; BOOCOCK, D.G. B.. Phase distributions of alcohol, glycerol, and catalyst in the transesterification of soybean oil. Journal of American Oil Chemists Society, v. 83, p.10471052, 2006. 